Original Paper http://ajol.info/index.php/ijbcs http://indexmedicus.afro.who.int

\title{
Perception des producteurs et impact des facteurs socio-économiques sur la connaissance des mycotoxines du maïs en stockage au Bénin
}

\author{
Rafiatou BA ${ }^{1,2}$, Nelly M. F MONTEIRO ${ }^{3}$, Ursula HOUNGUE ${ }^{2}$, Marcel T. DONOU \\ HOUNSODE $^{4}$, Fernand GBAGUIDI ${ }^{2}$ et Lamine BABA-MOUSSA ${ }^{1 *}$ \\ ${ }^{1}$ Laboratoire de Biologie et de Typage Moléculaire en Microbiologie ; Département de Biochimie et de \\ Biologie Cellulaire, Faculté des Sciences et Techniques, Université d'Abomey-Calavi, Bénin. \\ ${ }^{2}$ Laboratoire de Pharmacognosie et des Huiles essentielles, Centre Béninois de Recherche Scientifique et \\ Technique (CBRST), Bénin. \\ ${ }^{3}$ Laboratoire de Nutrition et Sciences Alimentaires, Département de Nutrition et Sciences Alimentaires, \\ Faculté des Sciences Agronomiques, Université d'Abomey-Calavi, Bénin. \\ ${ }^{4}$ Laboratoire de Biomathématiques et d'Estimations Forestières Faculté des Sciences Agronomiques, \\ Université d'Abomey-Calavi, Bénin. \\ *Auteur correspondant ; E-mail: laminesaid@yahoo.fr, Tel: + (229) 97123468
}

\section{REMERCIEMENTS}

Les auteurs adressent leurs remerciements au Projet de Productivité Agricole en Afrique de l'Ouest $(P P A A O)$ du Bénin pour avoir mis à leur disposition des moyens financiers pour la réalisation de la présente étude.

\section{RESUME}

Au Bénin, plusieurs technologies sont utilisées pour la conservation du maïs. Ces technologies utilisées ne contrôlent pas toujours les pertes fongiques enregistrées. L'objectif de l'étude est d'évaluer l'impact des facteurs socio-économiques des producteurs sur les technologies de prévention des pertes fongiques et leur niveau de connaissance des mycotoxines du maïs en stockage. Pour ce faire, une enquête a été réalisée dans sept zones agro-écologiques du Bénin. Au total, sept technologies de stockages du maïs ont été inventoriées (de A à G). La technologie B est non seulement présente dans toutes les zones parcourues mais aussi, elle est fortement représentée dans les zones III, IV et VIII avec des proportions respectives de $51,33 \%$; $62,86 \%$ et $69,83 \%$. La population agricole ayant un niveau secondaire (27\%) et les alphabétisés (15\%) ont la chance de connaître les mycotoxines. Les enquêtés $(100 \%)$ ne connaissent pas le danger lié à la consommation du maïs contaminé et n'ont jamais reçu une formation sur les mycotoxines. L'observation de la couleur verte $(61 \%$ des enquêtés) et la couleur noire ( $28 \%$ des enquêtés) témoigne bien de la persistance des mycotoxines dans le maïs en stock au Bénin.

(C) 2016 International Formulae Group. All rights reserved.

Mots clés : Technologie, conservation, céréales, contamination, moisissures. 


\title{
Perception of maize producers in Benin and impact of the socio-economic factors on the knowledge of the mycotoxins of maize in storage
}

\begin{abstract}
In Benin, various technologies are used for the conservation of the maize. Those technologies don't often control the recorded fungal losses. The objective of the study is to value the impact of the socioeconomic factors of producers on technologies of fungal loss prevention and their level of knowledge of mycotoxins of the maize in storage. A survey has been achieved in seven agro - ecological zones of Benin. Thus, a total of seven maize storage technologies were inventoried (A to G). The use of technology B is not only apparent in all the zones covered but it is also significantly used in zones III, IV and VIII in the respective proportions of $51.33 \% ; 62.86 \%$ and $69.83 \%$. The agricultural population, i.e. those with secondary education $(27 \%)$ and the literates $(15 \%)$ happen to have some knowledge about mycotoxins. People investigated $(100 \%)$ do not have knowledge of the danger associated with the consumption of contaminated maize and have never received training on mycotoxins. The observation of a green colour (by $61 \%$ of respondents) and black colour (by $28 \%$ of respondents) is testimony to the persistence of mycotoxins in maize under storage in Benin.
\end{abstract}

(C) 2016 International Formulae Group. All rights reserved.

Keywords: Technology, conservation, cereals, contamination, moulds.

\section{INTRODUCTION}

Pour de nombreux pays en voie de développement, les céréales représentent l'essentiel du régime alimentaire des populations rurales généralement à faibles revenus (Guèye et al., 2011). Parmi ces céréales, le maïs est une denrée de base pour les Africains. Cette céréale est cultivée dans sept sur huit zones agro-écologiques dont dispose le Bénin. Il fait donc l'objet d'importantes transactions nationales et régionales occupant ainsi une place de choix, contrairement au sorgho et au mil. Cependant, la production du maïs est confrontée à des contraintes d'ordre pédoclimatiques, socioéconomiques et biotiques (Antoine et al., 2013). La résolution progressive de ces contraintes et l'amélioration de la production peuvent s'avérer inutiles en l'absence d'un système adéquat de conservation post-récolte. Les récoltes, lorsqu'elles sont conservées dans les conditions inadéquates, en général sont attaquées par les insectes, les rongeurs et les moisissures. Les technologies de préservation du maïs incluant pratiques, produits chimiques et plantes insecticides sont utilisées par les producteurs dans la réduction des pertes physiques occasionnées par les insectes et les rongeurs. Contrairement aux pertes chimiques provoquées par les mycotoxines, aucune technique ni plante fongique n'est encore divulguée pour la réduction voire l'élimination de ces dernières. Pour Guèye et al. (2012), les moisissures occasionnent des diminutions de poids bien plus faibles que les insectes et constituent de ce fait, des facteurs d'altération qualitative à considérer. Par ailleurs la non adoption des systèmes de conservation adéquats par les producteurs expose les céréales en particulier le maïs aux problèmes de contamination chimiques (Fandohan et al., 2005). Chaque année, les cas de contamination des grains ou épis de maïs sont recensés par les producteurs. Cette contamination se traduit le plus souvent par des changements de couleur du maïs. Les moisissures réduisent la qualité des grains en produisant des mycotoxines. Ces infections fongiques ne doivent pas être mésestimées car elles peuvent être sévères et parfois entraîner des maladies graves, voire fatales. En effet, la présence des mycotoxines dans le maïs en stock, représente un risque important pour la santé des humains et des animaux qui en consomment. Chez les animaux, elles peuvent causer des effets néfastes sur le système immunitaire, la reproduction et la performance en général. Les dégâts occasionnés incluent la perte de poids, une diminution de la qualité du grain (Rajendran, 2002), et quelquefois une perte du pouvoir germinatif (Dabiré et al., 2008). L'activité métabolique des insectes crée un milieu favorable au développement des micro- 
organismes produisant des toxines à l'instar des champignons aflatoxinogènes du genre Aspergillus (Lamboni et al., 2009). Ces pertes chimiques peuvent s'expliquer par le nonrespect des techniques appropriées et les conditions climatiques aggravant l'action des insectes (Antoine et al., 2013 ). Au Bénin, les études de Fandohan et al. (2005) ont montré une prévalence de plus de $70 \%$ d'attaque par principalement des Fusarium sp. et des Aspergillus sp. Ces pertes chimiques occasionnées par la contamination des mycotoxines réduisent la qualité du grain et rendent le maïs impropre à la consommation. De ce fait, les pertes fongiques constituent un problème crucial pour les producteurs de maïs au Bénin. Il est donc nécessaire de ressortir parmi les différentes technologies de stockage celle donnant un faible taux voire une absence de grains contaminés. La présente étude a pour objectif de mieux comprendre la perception paysanne sur les pertes fongiques et l'impact des facteurs socio-économiques sur la connaissance des moisissures (mycotoxines) du maïs en conservation au Bénin. De façon spécifique, une enquête a été réalisée. Dans un premier temps, nous avons évalué l'impact des facteurs socioéconomiques sur le niveau de connaissance des moisissures du maïs en conservation; dans un deuxième temps, les usages des grains moisis et dans un troisième temps, nous avons ressorti les technologies de préservation du maïs fréquemment rencontrées et la perception des producteurs et productrices de maïs par rapport aux pertes fongiques.

\section{MATERIEL ET METHODES Cadre d'étude}

L'étude s'est déroulée dans sept zones agro-écologiques du Bénin. Il s'agit des zones II, III, IV, V, VI, VII et VIII. Ces zones ont été choisies suivant le rang qu'occupe le maïs dans les différentes cultures. Dans chacune des zones sélectionnées, deux communes ont été prises en compte suivant l'effectif de la population agricole et la quantité du maïs produite (Tableau 1).

\section{Echantillonnage}

La taille de l'échantillon a été déterminée par une approximation normale de la variable binomiale de Dagnelie (1998). Afin d'avoir une idée du nombre de producteurs et de productrices de maïs, nous avons estimé que $3 \%$ des producteurs de chacune des communes produisent du maïs. L'enquête a pris en compte 1011 producteurs et productrices de maïs dont 366 femmes.

\section{Collection des données sur les technologies de stockages du maïs}

Elle a consisté dans un premier temps à tester le questionnaire sur quatre producteurs parlant des langues locales différentes. Ceci a permis d'adapter le questionnaire aux réalités du terrain. Les langues explorées étaient : Goun, Dendi, Mina et Bariba. Dans un second temps, le questionnaire a été adressé de façon individuel aux producteurs et productrices de maïs selon leur disponibilité. Ceci a permis de ressortir les différentes étapes intervenant dans chaque technologie de stockage du maïs, d'évaluer le niveau de connaissance des producteurs sur la contamination du maïs par les mycotoxines, sur le danger lié à la consommation des grains moisis sur les pratiques, produits chimiques et locales utilisés pour la prévention des pertes fongiques. L'enquête s'est déroulée dans 14 communes du 12 août au 29 octobre 2014. Il s'agit des communes de Kandi, Banikoara, Kalalé, Djougou, Natittingou, Djakotomey, Abomey-Calavi, Nikki, Aplahoué, Lokossa, Tchaourou, Pobè, Lalo et Dangbo. Le choix des communes s'est fait de concert avec les cadres du Centre d'Action Régional pour le Développement Rural (CARDER).

\section{Analyse statistique \\ Le logiciel R.3.1.2 a été utilisé pour calculer la fréquence d'utilisation des technologies de stockage du maïs et le niveau de connaissance sur la contamination par les mycotoxines. Le logiciel Exel a permis de construire les graphes.}

\section{RESULTATS}

\section{Caractéristiques socio-économiques et démographiques des producteurs et productrices de maïs}

L'étude des caractéristiques socioéconomiques et démographiques des enquêtés nous a permis de répartir la population selon 
plusieurs critères : l'âge, le sexe, le statut social et le niveau d'instruction.

\section{Répartition de la population agricole en fonction du sexe et de l'âge}

Le Tableau 2 montre la répartition des producteurs en fonction de l'âge. L'analyse de la moyenne effectuée sur l'âge donne une probabilité $\mathrm{p}=0,0001<0,05$. Il existe une différence significative au seuil de $5 \%$ entre les âges des populations enquêtées. Dans les zones II, III, IV, V, VII et VIII, la moyenne d'âge n'est pas significativement différente au seuil de 5\%. Quant à la zone VI, l'âge moyen diffère significativement (Tableau 2).

En ce qui concerne la répartition des enquêtés en fonction du sexe, $66 \%$ sont de sexe masculin (M) et $34 \%$ sont de sexe féminin (F) (Figure 1).

Répartition des enquêtés en fonction du niveau d'instruction et la connaissance des mycotoxines

Une sélection pas à pas effectuée sur les variables indépendantes montre que la modélisation du niveau de connaissance des mycotoxines ne peut être fonction que du niveau d'instruction avec un critère d'information d'AKAIKE relativement faible $(\mathrm{AIC}=88,54)$ en comparaison avec celui du model initial (AIC=93,79). La modélisation de la connaissance des aflatoxines en fonction du niveau d'instruction a donné les résultats suivant le Tableau 3.

Les probabilités trouvées pour les variables indépendantes sont inférieures à 0,05 . Ce qui signifie que ces variables ont un impact positif sur la connaissance des aflatoxines de la population enquêtée.

\section{Répartition des enquêtés en fonction de l'utilisation des grains moisis}

Pour toutes les technologies recensées, les enquêtés (100\%) enregistrent des pertes fongiques. Deux principales couleurs ont été observées parmi les grains moisis (Figure 2). Nous avons la couleur verte remarquée par $61 \%$ des enquêtés et la couleur noire par $28 \%$ des enquêtés. Les deux couleurs ont été observées à la fois par $11 \%$ des producteurs. Quant à l'usage des grains moisis, les grains noirs sont automatiquement jetés ou brûlés, mais les grains verts servent à d'autres fins (Figure 3).

\section{Proportion d'utilisation des Technologies de stockage du maïs par zone agro- écologique}

Pour les sept zones agro-écologiques parcourues, nous avons recensé sept méthodes de stockage différentes. Dans chaque zone, nous avons en moyenne cinq technologies de stockage de maïs. Cette variabilité des méthodes de stockages peut s'expliquer par la non-assistance des agents du CARDER pendant la conservation du maïs.

La variation des technologies de stockage et de conservation du maïs se trouve au niveau des structures de stockage, des pratiques, du conditionnement, du temps de séchage, des produits chimiques et des plantes locales utilisés (Figure 4). Ainsi, pour la Technologie A, nous avons : la maturation ; le séchage solaire sur pied; la récolte ; l'égrenage; application des produits chimiques (nom et quantité du produit chimique varie selon le producteur); stockage dans les sacs en Polyéthylène puis entreposage dans les magasins ou chambres d'habitation. Cette technologie est réalisée par $90 \%$ des producteurs du Nord et $80 \%$ des producteurs du Sud Bénin (Hinnou et Aloukoutou 2011). La Technologie B est similaire à la Technologie $\mathrm{A}$ à la différence que, après égrenage, l'application des produits chimiques n'intervient pas. La Technologie $\mathrm{E}$ : après maturation, nous avons le séchage solaire, l'égrenage et le stockage dans le grenier. La Technologie D : à la technologie E, s'ajoute l'utilisation des plantes locales dans le grenier. Cette dernière est également décrite par Guèye et al. (2012) qui est couramment utilisée au Sénégal. Pour Hinnou et Aloukoutou (2011), l'utilisation des Technologies D et E ne sont pas conseillées aux grands producteurs de maïs. En effet, à cause des grandes superficies emblavées et de l'importance de la récolte, le stockage dans les greniers exigerait que le producteur en construise beaucoup, ce qui ne l'avantage pas. La Technologie F : après maturation, séchage solaire, récolte, égrenage, les grains de maïs sont stockés dans des paniers, fûts, bidon ou étaler sur le plafond avec utilisation des 
plantes locales (variant selon le producteur). L'utilisation de la Technologie F permet la non utilisation de produits de conservation mais de limiter les taux de pertes post récoltes (Hinnou et Aloukoutou, 2011). Enfin, la Technologie G, après la maturation, nous avons le séchage solaire et la récolte des épis de maïs. Ces épis non despathés sont disposés en vrac au sol dans la chambre pour leur stockage. Cette technique est utilisée par $20 \%$ des petits producteurs au Nord et 10 à $30 \%$ au Sud du Bénin (Hinnou et Aloukoutou, 2011).

Durée de conservation et de séchage des grains de maïs en fonction des zones agro écologiques

La durée de conservation et de séchage des grains de maïs varient d'une zone agroécologique à une autre $(\mathrm{P}<5 \%)$. Elle évolue d'environ 3 à 10 mois pour la conservation et oscille entre 1 et 4 mois pour le séchage. En effet, les zones agro-écologiques couvrent les départements du Sud et du Nord. La différence observée peut s'expliquer par la divergence existante dans le nombre de saisons sèche et pluvieuse entre ces zones (Tableau 4).

Répartition de la durée de conservation et de séchage en fonction des technologies

La durée de conservation $(\mathrm{P}=1.017 \mathrm{e}-07$ $<0,05)$ et de séchage $(\mathrm{P}=4.976 \mathrm{e}-07<0,05)$ varient d'une technologie à une autre. Ceci peut être lié aux phénomènes socioéconomiques des enquêtés (Tableau 5). En effet, lorsque le besoin se fait sentir, le producteur peut soit réduire le temps de séchage, soit rompre la conservation ou vendre son maïs (Tableau 5).

Tableau 1 : Coordonnées géographiques des communes enquêtées.

\begin{tabular}{ccccc}
\hline $\begin{array}{c}\text { Zones Agro- } \\
\text { écologiques }\end{array}$ & Départements & Communes & $\begin{array}{c}\text { Latitude de la } \\
\text { commune }\end{array}$ & $\begin{array}{c}\text { Longitude de la } \\
\text { commune }\end{array}$ \\
\hline Zone II & Alibori & Banikoara & $11^{\circ} 17^{\prime} 54^{\prime \prime}$ nord & $2^{\circ} 56^{\prime} 18^{\prime \prime}$ est \\
& & Kandi & $11^{\circ} 08^{\prime} 03^{\prime \prime}$ nord & $2^{\circ} 26^{\prime} 18^{\prime \prime}$ est \\
Zone III & Borgou & Nikki & $9^{\circ} 55^{\prime} 60^{\prime \prime}$ nord & $3^{\circ} 12^{\prime} 30^{\prime \prime}$ est \\
& & Kalale & $10^{\circ} 17^{\prime} 24^{\prime \prime}$ nord & $3^{\circ} 22^{\prime} 54^{\prime \prime}$ est \\
Zone IV & Donga & Djougou & $9^{\circ} 42^{\prime}$ nord & $1^{\circ} 40^{\prime} 0^{\prime \prime}$ est \\
& Atacora & Natitingou & $10^{\circ} 18^{\prime} 46^{\prime \prime}$ nord & $1^{\circ} 23^{\prime} 19^{\prime \prime}$ est \\
Zone V & Couffo & Aplahoué & $6^{\circ} 56^{\prime} 32^{\prime \prime}$ nord & $1^{\circ} 40^{\prime} 25^{\prime \prime}$ est \\
Zone VI & Atlantique & Abomey- calavi & $6^{\circ} 26^{\prime} 54^{\prime \prime}$ Nord & $2^{\circ} 21^{\prime} 20^{\prime \prime}$ est \\
& Couffo & Djakotomey & $6^{\circ} 54^{\prime}$ nord & $1^{\circ} 43^{\prime} 0^{\prime \prime}$ est \\
Zone VII & Plateau & Pobè & $6^{\circ} 58^{\prime} 60^{\prime \prime}$ nord & $2^{\circ} 39^{\prime} 49^{\prime \prime}$ est \\
& Couffo & Lalo & $6^{\circ} 55^{\prime} 26^{\prime \prime}$ nord & $1^{\circ} 53^{\prime} 11^{\prime \prime}$ est \\
Zone VIII & Mono & Lokossa & $6^{\circ} 37^{\prime} 60^{\prime \prime}$ nord & $1^{\circ} 43^{\prime} 0^{\prime \prime}$ est \\
& Ouémé & Dangbo & $6^{\circ} 34^{\prime} 42^{\prime \prime}$ nord & $2^{\circ} 33^{\prime} 28^{\prime \prime}$ est \\
\hline
\end{tabular}

Tableau 2: Population agricole en fonction de l'âge.

\begin{tabular}{|c|c|c|c|}
\hline Variable & Zones & Moyenne & Probabilité \\
\hline \multirow{7}{*}{ Age } & II & $41,62 \pm 0,716^{\mathrm{a}}$ & \multirow{7}{*}{0,0001} \\
\hline & III & $39,04 \pm 0,957^{\mathrm{a}}$ & \\
\hline & IV & $37,43 \pm 1,07^{\mathrm{a}}$ & \\
\hline & V & $39,639 \pm 0,812^{\mathrm{a}}$ & \\
\hline & VI & $47,15 \pm 1,08^{\mathrm{b}}$ & \\
\hline & VII & $40,43 \pm 1,07^{\mathrm{a}}$ & \\
\hline & VIII & $43,866 \pm 0,815^{\mathrm{a}}$ & \\
\hline
\end{tabular}


Tableau 3 : Niveau d'instruction en fonction de la connaissance des mycotoxines.

\begin{tabular}{lccc}
\hline Références & Variables & Rapport de chance (\%) & Probabilité \\
\hline Non Scolarisé & Alphabétisé & 15,04 & 0,003544 \\
& Primaire & 1,27 & 0,835345 \\
& Secondaire & 26,91 & 0,000473 \\
\hline
\end{tabular}

Tableau 4: Durée de conservation et de séchage en fonction des zones agro-écologique.

\begin{tabular}{lccc}
\hline Variables & Zones & Moyenne & Probabilité \\
\hline \multirow{4}{*}{ Conservation } & II & $10,368 \pm 0,108$ & $2.2 \mathrm{e}-16$ \\
III & $5,313 \pm 0,109$ & \\
IV & $6,143 \pm 0,201$ & \\
V & $7,079 \pm 0,102$ & \\
VI & $2,9699 \pm 0,0237$ & \\
VII & $7,496 \pm 0,168$ & \\
VII & $8,212 \pm 0,127$ & \\
\hline Séchage & II & $2,227 \pm 0,0876$ & \\
& III & $2,9467 \pm 0,0508$ & \\
IV & $3,8143 \pm 0,0796$ & \\
& V & $3,8143 \pm 0,0417$ & \\
& VI & $1,1053 \pm 0,0468$ & \\
VII & $1,0226 \pm 0,0129$ & \\
& VII & $1,0615 \pm 0,018$ & \\
\hline
\end{tabular}

Tableau 5 : Durée de conservation et de séchage en fonction des technologies.

\begin{tabular}{lccc}
\hline Variables & Technologie & Moyenne & Probabilité \\
\hline & A & $6,948 \pm 0,166$ & $1.017 \mathrm{e}-07$ \\
B & $7,136 \pm 0,126$ & \\
Conservation & C & $6,7 \pm 0,478$ & \\
& D & $6,803 \pm 0,192$ & \\
E & $7,558 \pm 0,192$ & \\
F & $3,333 \pm 0,333$ & \\
G & $4,33 \pm 1,33$ & \\
Séchage & A & $1,8913 \pm 0,067$ & $4.976 \mathrm{e}-07$ \\
B & $1,9386 \pm 0,059$ & \\
C & $1,65 \pm 0,141$ & \\
D & $1,4904 \pm 0,0613$ & \\
E & $1,9156 \pm 0,0879$ & \\
& F & $1 \pm 0$ & \\
G & $1,333 \pm 0,3333$ & \\
\hline
\end{tabular}


R. BA et al. / Int. J. Biol. Chem. Sci. 10(1): 155-166, 2016

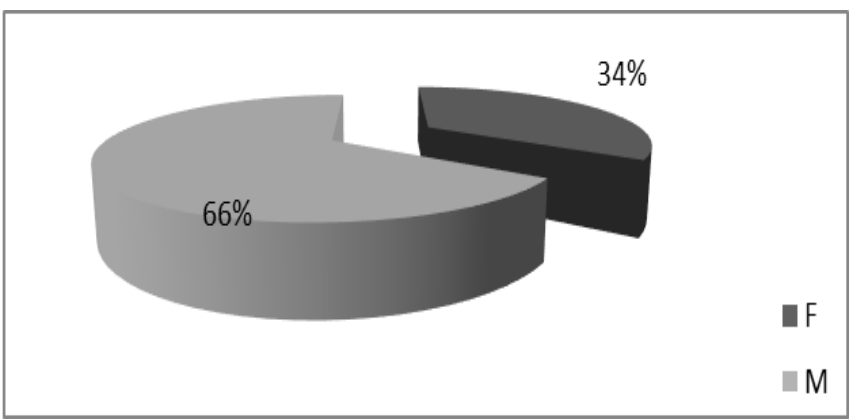

Figure 1: Répartition de la population agricole en fonction du sexe. $\mathrm{F}$ : féminin ; $\mathrm{M}$ : masculin.

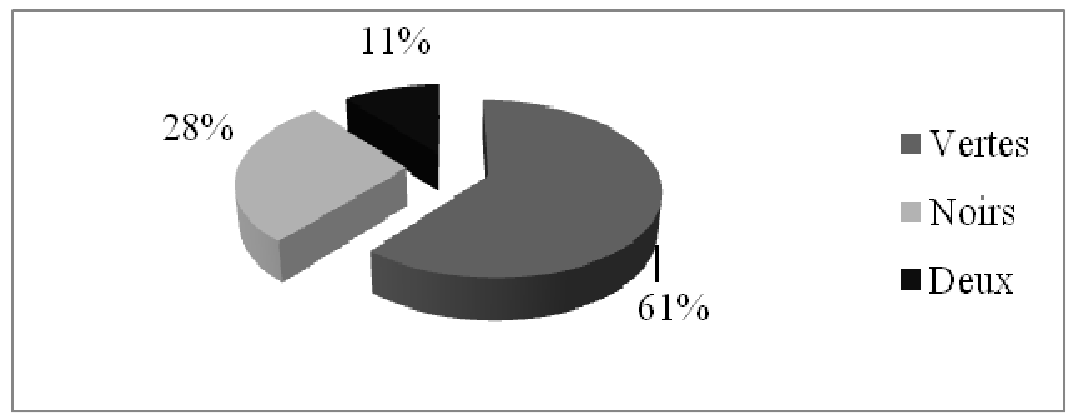

Figure 2 : Proportion d'apparition des couleurs en fonction des producteurs.

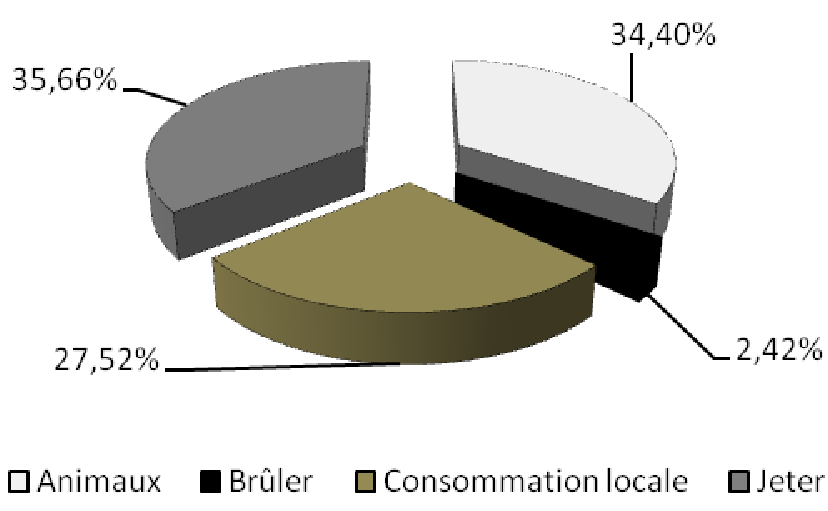

Figure 3 : Différents usages des grains de maïs moisis. 


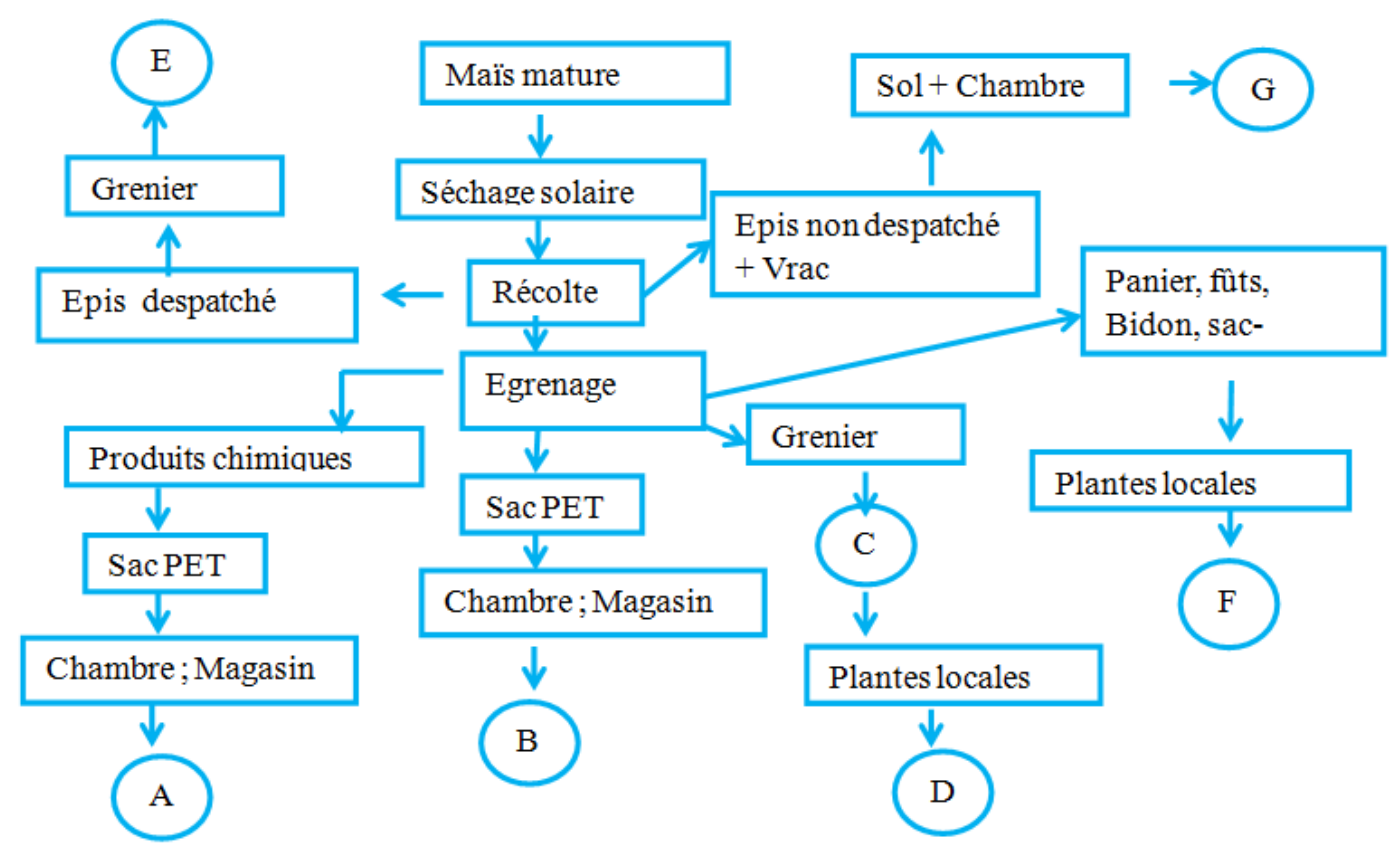

Figure 4 : Différentes Technologies de stockage du maïs.

Les technologies A, B, D et $\mathrm{E}$ sont présentent dans toutes les zones parcourus alors que la technologie $\mathrm{C}$ est présente dans les zones II, III, VI et VIII. Les technologies F et G sont respectivement présents dans les zones IV et VI.

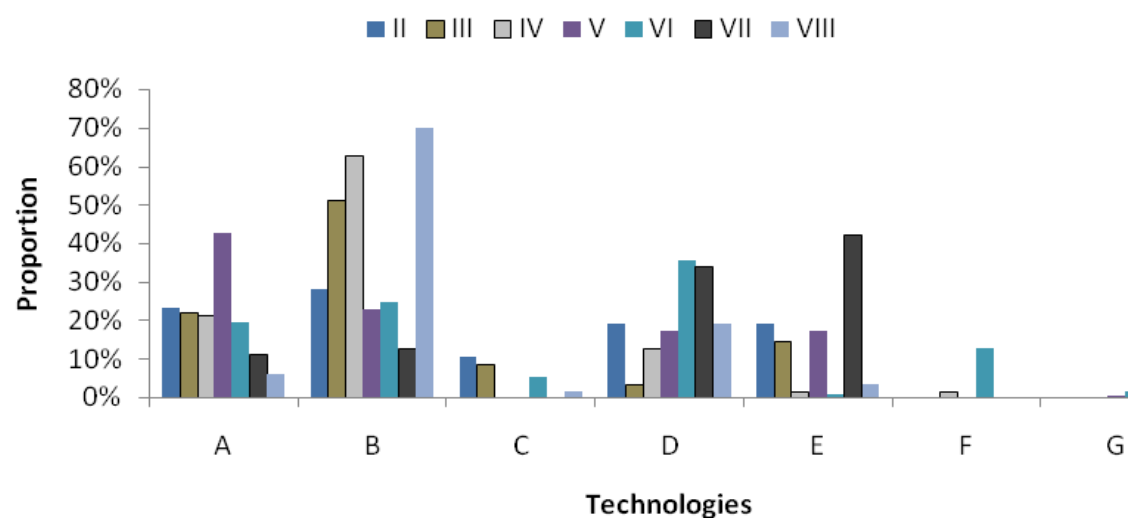

Figure 5 : Technologies de stockage du maïs en fonction des zones agro-écologiques.

\section{DISCUSSION}

Cette étude a permis de ressortir le niveau de connaissance des producteurs et productrices de maïs sur les moisissures et les technologies utilisées par ces derniers dans le cadre de la prévention fongique. Pour toutes les zones-agroécologiques parcourues, le maïs est conservé aussi bien par les hommes (66\%) que les femmes (34\%) (Figure 1). Ce résultat est en adéquation avec les résultats de recherches de Sibiri et Kaboré (2014) qui ont montré que la production nationale du maïs en Afrique de l'ouest cache d'importantes disparités dans la production selon le genre. Ce faible taux de conservation du maïs par les femmes s'explique par le fait que le niveau de 
production et de rendement du maïs obtenu par les femmes reste largement en deçà de celui obtenu par les hommes, ceci dénote de la faible accessibilité des femmes à la terre (Sohinto et Aïna, 2010). Ces observations sont contraires à ceux de Gansou et al. (2000) et de Hell et al. (2000) qui ont montré que les femmes enregistrent pour le stockage du maïs des niveaux de perte allant jusqu'à $75 \%$ lorsqu'elles n'appliquent aucun traitement phytosanitaire. Ceci confirme bien nos observations faites au cours de l'enquête ou les femmes, s'intéressent de plus en plus à la conservation d'autres cultures telles que le riz, le haricot, le soja. Car selon elles, ces cultures se conservent beaucoup plus facilement (faible moyen financier) avec un faible taux en pertes post récoltes. Nous notons également une grande variabilité entre les âges des enquêtés d'une zone à une autre avec une moyenne se situant entre (37 - 47) ans (Tableau 2). La connaissance des mycotoxines n'est liée ni à l'âge ni au sexe mais plutôt au niveau d'instruction. Alors $27 \%$ des instruits secondaires et $15 \%$ des alphabétisés ont la chance de connaître les mycotoxines alors que les instruits primaires et les non scolarisés n'ont pratiquement pas la chance de connaître ce que c'est que les mycotoxines (Tableau 3). Aussi, $100 \%$ des enquêtés ignorent le danger lié à la consommation du maïs contaminé par les mycotoxines, et n'ont jamais reçu une formation sur ces dernières. Ce résultat est contraire à celui de Allomasso (2006) qui a montré au cours d'une enquête que $82 \%$ des répondants reconnaissent que les aliments moisis sont dangereux et que la raison fondamentale réside dans les maladies qu'ils provoquent. Par ailleurs, les premiers signes d'infection des grains après la récolte se manifestent par des changements de couleur. Au Bénin, plus de 30\% des grains de maïs sont contaminés avec l'aflatoxine (Allogni et al., 2010). L'observation de la couleur verte par $61 \%$ des enquêtés et la couleur noire par $28 \%$ des enquêtés témoigne bien de la persistance des moisissures dans le maïs en stock au Bénin. Ce résultat est comparable à celui de Guèye et al. (2012) qui affirment que $56 \%$ des enquêtés ont révélé la présence de moisissures dans les stocks de maïs au Sénégal. Quant à l'usage des grains moisis, les grains noirs sont automatiquement jetés ou brûlés, mais les grains verts servent à d'autres fins. Ces derniers sont soit consommés localement ou donnés aux animaux. Ainsi, $35,66 \%$ des producteurs de maïs jettent leurs grains moisis. En effet, ceux-ci ne disposant pas d'animaux, n'arrivent plus à utiliser les grains moisis. 34,40\% des enquêtés par contre nourrissent leurs bétails de ces grains. Certains producteurs $(27,52 \%)$ consomment ces grains malgré le changement de couleur (verdâtre). Selon ces derniers, ces grains sont toujours comestibles vu qu'ils n'ont pas encore été rongés par les insectes et qu'il s'agit d'une simple humidification. Cependant, 2,42\% des producteurs brûlent ces derniers. Ceci s'explique par le niveau de connaissance de ces producteurs sur le danger lié à la consommation du grain infecté par les moisissures. Aussi, les producteurs (100\%) affirment n'avoir utilisé aucun produit ni plante locale destiné à la prévention des pertes fongiques. En effet, les résultats de l'enquête montrent que pour toutes les zones parcourues nous avons sept technologies différentes de stockage de maïs couramment utilisées par les enquêtés. Pour une même zone agroécologique, nous avons en moyenne cinq méthodes différentes de stockage de maïs. Les différences entre ces méthodes de stockage se situent au niveau du temps de maturation, de séchage, des pratiques, des produits chimiques, des plantes locales, du conditionnement et des lieux de stockages du maïs (Figure 4). Ceci témoigne bien de la variabilité des technologies de stockage du maïs. Cette variabilité des méthodes de stockages peut s'expliquer par la nonassistance des agents du CARDER pendant la conservation du maïs. La variation des technologies se trouve au niveau des structures de stockage, des pratiques, du conditionnement, du temps de séchage, des produits chimiques et des plantes locales 
utilisés. D’une façon générale, les technologies de stockage recensées sont présentes dans les zones parcourues mais les proportions d'utilisation de ces dernières varient d'une zone à une autre. Les méthodes A $(22,03 \%)$, B $(37,45 \%), D(19,92 \%)$ et E $(14,75 \%)$ sont les plus utilisées par les producteurs et productrices de maïs au regard des méthodes C $(3,83 \%), \mathrm{F}(1,72 \%)$ et $\mathrm{G}$ $(0,29 \%)$. Dans les zones II, III, IV et VIII, la méthode $\mathrm{B}$ est non seulement présente mais elle est fortement représentée avec des proportions respectives de $28,22 \%$; $51,33 \%$; $62,86 \%$ et $69,83 \%$. La forte utilisation de cette méthode peut s'expliquer par le fait qu'elle est très facile à réaliser pour une grande production et n'utilise aucun produit chimique pendant la conservation. De plus, la durée de conservation de cette méthode est courte (2 à 3 mois). Par contre, dans les zone V; VI et VII, ce sont les méthodes A $(42,59 \%)$, D $(35,34 \%)$ et E $(42,11 \%)$ qui sont considérablement représentées. Le fort taux d'usage de la technologie A dans ces zones peut être dû au fait que le maïs est conservé sur une longue période par les gros producteurs (possédant des moyens pour l'achat des produits chimiques). Pour Hinnou et Aloukoutou (2011), l'utilisation de la technologie A s'explique par sa facilité de réalisation. L'utilisation considérable de la technologie E s'explique par le fait que c'est une méthode pratiquée par les petits producteurs et productrices de maïs. Cette dernière n'a recours ni aux plantes locales ni aux produits chimiques. Des observations similaires ont été faites par Guèye et al. (2012) au Sénégal. En effet, ils ont recensé les mêmes technologies de stockage chez les producteurs et productrices de maïs. Des résultats issus des recherches de ces derniers, nous avons les technologies A, B, D et E qui sont aussi fortement utilisées. Les taux d'utilisation des technologies F $(1,72 \%)$ et $\mathrm{G}$ $(0,29 \%)$ obtenus sont très inférieurs à ceux trouvés par Hinnou et Aloukoutou (2011). Pour ces chercheurs, les enquêtés (20\%) utilisent la technologie $F$ et la technologie $G$
(10 à 30\%). Le faible taux d'utilisation des technologies $\mathrm{F}$ et $\mathrm{G}$ est dû au fait que ce sont des méthodes héritées par certains enquêtés très attachés à la tradition. Pour Adégbola et al. (2011), plusieurs facteurs peuvent expliquer les réticences observées chez les producteurs. Parmi ces facteurs, on peut citer entre autres, la rareté des matériaux primaires, le coût de construction élevé et l'environnement socio-économique du producteur (Mendola, 2007). Par ailleurs, le faible taux d'utilisation des technologies $\mathrm{F}$ et $\mathrm{G}$ s'explique par le manque de moyens financiers des producteurs (Hinnou et Aloukoutou, 2011). En effet, vu que le petit stock de maïs est destiné à etre utilisé pour une courte durée, les producteurs ne trouvent plus l'importance de se déranger pour la conservation. Pour toutes ces technologies, les producteurs et productrices de maïs enregistrent toujours des pertes fongiques. L'analyse microbiologique des sept technologies de stockage recensées permettra d'identifier la technologie aboutissant à un faible taux de grains contaminés par les mycotoxines. Ceci permettra non seulement de garantir la sécurité sanitaire des consommateurs, mais aussi de disposer d'une méthode efficace pour faire une meilleure conservation des céréales.

\section{CONFLIT D'INTERET}

Les auteurs déclarent qu'il n'existe aucun conflit d'intérêt relatif à cet article.

\section{CONTRIBUTION DES AUTEURS}

$\mathrm{RB}$ a élaboré le sujet, et a conduit la recherche, la collecte des données sur le terrain, la saisie des données collectées pour analyse statistique et la rédaction du manuscrit avec UH; NMFM a conduit la recherche et a contribué à l'écriture du manuscrit; MTDH a réalisé l'analyse statistique des données collectées sur le terrain et a contribué à l'écriture du manuscrit; FG et LB-M participé à l'élaboration du sujet, la conduite de la recherche et la contribution à l'écriture du manuscrit. 


\section{REMERCIEMENTS}

Nous remercions l'Agence Universitaire de la Francophonie pour son apport scientifique. Nous sommes aussi très reconnaissants à l'égard des conseillers agricoles, des producteurs et productrices de maïs pour leur disponibilité.

\section{REFERENCES}

Adégbola PY, Arouna A, Ahoyo NRA. 2011. Acceptabilité des structures améliorées de stockage du maïs au Sud-Bénin. Bulletin de la Recherche Agronomique du Bénin Numéro spécial 2 Aspects économiques du stockage et de la conservation du maïs au Sud-Bénin.

Allogni WN, Coulibaly ON, Hell K, Mensah GA. 2010. Liens entre la contamination des produits maïsicoles à l'aflatoxine et la pauvreté au Bénin. Bulletin de la Recherche Agronomique du Bénin, 68.

Allomasso R, Coulibaly O, Glitho I, Hell K. 2006. Principaux facteurs affectant l'état nutritionnel et de santé des enfants exposés à l'aflatoxine: Application du madel Probit. Bulletin de la Recherche Agronomique du Bénin, 53.

Antoine W, Marcelin Y, Clémentine L, Dabiré B, Malick N. 2013. Conservation postrécolte des céréales en zone sudsoudanienne du Burkina Faso: Perception paysanne et évaluation des stocks. International Journal of Biological and Chemical Sciences, 7(3): 1157-1167. DOI: http://dx.doi.org/10.4314/ ijbcs.v7i3.22

Dabiré C. 2001. Etude de quelques paramètres biologiques et écologiques de Clavigralla tomentosi collis STAL, (Hemiptera : Coreidae) punaise suceuse suceuses des gousses du niébé (Vigna unguiculata L.) dans une perspective de lutte durable contre l'insecte au Burkina. Thèse de Doctorat d'état, Université de Cocody, 179p.

Fandohan P, Gnonlonfin B, Hell K, Marasa WFO, Wingfield MJ. 2005. Impact of indigenous storage systems and insect infestation on the contamination of maize with fumonisins. African Journal of Biotechnology, 5(7): 546-552.

Fandohan P, Ahouansou R, Houssou P, Hell K, Marasas WFO, Wingfield MJ. 2006. Impact of mechanical shelling and dehulling on Fusarium infection and fumonisin contamination in maize. Food Additives and Contaminants, 23(4): 415421. DOI: http://dx.doi.org/10.1080/ 02652030500442516.

Gansou G, Yabi M, Kiki E. 2000. Etude des Modes de «Vente à Terme» et de «Vente Précoce» des Récoltes du Maïs par les Producteurs. PADSA/DANIDA : Cotonou, Bénin ; 43.

Goubalan E. 2011. Revue documentaire sur les filières agricoles et le crédit vivrier au Bénin : cas de la filière maïs. Rapport d'étude, 38p.

Guèye MT, Seck D, Wathelet J-P, Lognay G. 2011. Lutte contre les ravageurs des stocks de céréales et de légumineuses au Sénégal et en Afrique occidentale : synthèse bibliographique. Biotechnol. Agron. Soc. Environ., 15(1): 183-194.

Guèye TM, Seck D, Wathelet JP, Lognay G. 2012. Typologie des systèmes de stockage et de conservation du maïs dans l'est et le sud du Sénégal. Biotechnol. Agron. Soc. Environ., 16(1): 49-58.

Hell KKF, Cardwella MH, Setamou MP. 2000. The influence of storage practices on aflatoxin contamination in maize in four agroecological zones of Benin, West Africa. Journal of Stored Products Research, 36: 365-382. DOI: http://dx.doi.org/10.1016/S0022474X(99)00056-9.

Hinnou CL, Aloukoutou MA. 2011. Stockage et conservation du maïs au Bénin : Techniques efficaces et stratégies d'adoption. Rapport d'étude, 56p.

Lamboni Y, Hell K. 2009. Propagation of mycotoxigenic fungi in maize stores by post-harvest insect. Int. J. Trop. Insect Sci., 29(1): 31-39. 
Mendola M. 2007. Agricultural technology adoption and poverty reduction: A propensity score matching analysis for rural Bangladesh. Food Policy, 32: 372393. DOI: http://dx.doi.org/10.1016/ j.foodpol.2006.07.003.

Rahimi E, Bonyadian M, Rafei M, Kazemeini HR. 2009. Occurrence of aflatoxin M1 in raw milk of five dairy species in Ahvaz, Iran. Food Chem. Toxicol., 48: 129-131. DOI: http://dx.doi.org/10.1016/ j.fct.2009.09.028.

Rajendran S. 2002. Postharverst pest losses. In Encyclopedia of Pest Management, Pimentel D (ed). Marcel Dekker, Inc: New York ; 654-656.

Sibiri JO, Kaboré WT. 2014. Etude prospective sur les pertes post récoltes en
Afrique de l'ouest : cas de quelques pays côtiers et du sahel. Institut du sahel. Rapport définitif, 44p.

Sohinto D, Aïna M. 2010. Analyse économique et financière de cinq chaînes de valeurs ajoutées (CVA) de la filière maïs au Bénin. Rapport provisoire d'étude, 76p.

Sohinto D, Aïna M. 2011. Etude documentaire sur la filière maïs et ses chaînes de valeurs ajoutées au Bénin. Rapport provisoire, $135 \mathrm{p}$.

Wankpo E. 2010. Etat des lieux de l'exportation des produits vivriers et de son impact sur les petits producteurs dans la chaine de valeur: cas du maïs au Bénin. Rapport d'étude, 26p. 